\title{
COMPUTING SKEW LEFT BRACES OF SMALL ORDERS
}

\author{
VALERIY G. BARDAKOV, MIKHAIL V. NESHCHADIM, AND MANOJ K. YADAV
}

\begin{abstract}
We improve Algorithm 5.1 of [Math. Comp. 86 (2017), 2519-2534] for computing all non-isomorphic skew left braces, and enumerate left braces and skew left braces of orders up to 868 with some exceptions. Using the enumerated data, we state some conjectures for further research.
\end{abstract}

\section{INTRODUCTION}

A triple $(G,+, \circ)$, where $(G,+)$ and $(G, \circ)$ are (not necessarily abelian) groups, is said to be a skew left brace if

$$
g_{1} \circ\left(g_{2}+g_{3}\right)=\left(g_{1} \circ g_{2}\right)-g_{1}+\left(g_{1} \circ g_{3}\right)
$$

for all $g_{1}, g_{2}, g_{3} \in G$, where $-g_{1}$ denotes the inverse of $g_{1}$ in $(G,+)$. We call $(G,+)$ the additive group and $(G, \circ)$ the multiplicative group of the skew left brace $(G,+, \circ)$. A skew left brace $(G,+, \circ)$ is said to be a left brace if $(G,+)$ is an abelian group. The concept of left braces was introduced by Rump [18 in 2007 in connection with non-degenerate involutive set theoretic solutions of the quantum Yang-Baxter equations. Thereafter the subject received a tremendous attention of the mathematical community; see [3, 5, 19, 20, and the references therein. Interest in the study of set theoretic solutions of the quantum Yang-Baxter equations was intrigued by the paper 11 of Drinfeld, published in 1992.

Let $X$ be an arbitrary set and $R: X \times X \rightarrow X \times X$ a bijective map. Recall that the pair $(X, R)$ is said to be a set theoretic solution of the Yang-Baxter equation if

$$
R_{12} R_{23} R_{12}=R_{23} R_{12} R_{23}
$$

holds in the set of all maps from $X \times X \times X$ to itself, where $R_{i j}$ is just $R$ acting on the $i$ th and $j$ th components of $X \times X \times X$ and identity on the remaining one. Let us write

$$
R(x, y)=\left(\sigma_{x}(y), \tau_{y}(x)\right), x, y \in X
$$

with $\sigma_{x}$ and $\tau_{y}$ component maps from $X$ to itself.

A solution $(X, R)$ is said to be non-degenerate if the component maps $\sigma_{x}$ and $\tau_{y}$ are bijections on $X$ for all $x, y \in X$. It is said to be involutive if $R^{2}$ is the identity map. The study of non-degenerate set theoretic solutions of the quantum Yang-Baxter equations has been extensively taken up, e. g., [6, 9, 13, 16, 21] to mention a few.

The concept of skew left brace was introduced by Guarnieri and Vendramin 15 in 2017 in connection with non-involutive non-degenerate set theoretic solutions of the quantum Yang-Baxter equations. They invented an algorithm, by generalising a result of Bachiller [2] for computing all skew left braces of a given order. They themselves computed left braces and skew left braces of lot of groups upto order 120. Vendramin [22] extended the number upto 168 with some exceptions. All these computations are done using computer algebra systems MAGMA [4 and GAP [14] using the algorithm invented in [15]. For more work on skew braces see [7, 8, 17.

This article aims at filling up the gaps in the table produced in 22 to some extent and making further computations for larger orders. An ingenious observation on regular

2010 Mathematics Subject Classification. 16T25, 81R50, 20B40.

Key words and phrases. Skew left braces, Left braces, Regular subgroups, Yang-Baxter equation. 
subgroups of the holomorph of a given finite group allows us to improve the algorithm obtained in [15, which substantially enhances the performance of MAGMA computation. The improved algorithm, actually, avoids an expensive calculation in the existing algorithm. We compute the number of non-isomorphic left braces and skew left braces of orders upto 868 except certain cases (mainly when the order is a multiple of 32). These results settle [22, Problem 13] and [15, Problem 6.1]. The computations will help in building a database of left braces and skew left braces, which in turn will greatly enrich the library of solutions of the quantum Yang-Baxter equation. On the basis of our computation, we suggest some conjectures for further research.

It is striking that there are more than a million skew brace structures of order $2^{5}$ and more than 20 millions skew brace structures of order $3^{5}$. The reader will encounter many more surprises while going through the tables. We have used MAGMA on a computer with $3.5 \mathrm{GHz}$ 6-Core Intel Xeon E5 processor and 64 GB memory for these computations.

\section{Regular subgroups}

Let $G$ be a group, which acts on a set $X$. The action of an element $g \in G$ on an element $x \in X$ is denoted by $x^{g}$. A subgroup $H$ of $G$ is said to be action-closed if for each pair $(g, x) \in G \times X$, there exists an element $h \in H$ such that $x^{g}=x^{h}$. By $H$ - conjugacy class of $x \in G$, we mean $\left\{x^{h} \mid h \in H\right\}$. For $g, h \in G$, we write the conjugate of $g$ by $h$ as $g^{h}=h^{-1} g h$.

Let $G$ be a group and $\operatorname{Symm}(G)$ be the symmetric group on the set $G$. Recall that a subgroup $\mathcal{G}$ of $\operatorname{Symm}(G)$ is said to be regular if $\mathcal{G}$-action on $G$ is free and transitive. By a free action we here mean that for any element $g \in G$, its stabilizer in $\mathcal{G}$ is the trivial subgroup. Observe that when $G$ is finite, any regular subgroup of $\operatorname{Symm}(G)$ is of order $|G|$.

For a group $G, \operatorname{Hol}(G)$ denotes the holomorph of $G$, which is defined as the semidirect product of $G$ with $\operatorname{Aut}(G)$, the automorphism group of $G$. So

$$
\operatorname{Hol}(G):=\operatorname{Aut}(G) \ltimes G,
$$

where the product in $\operatorname{Hol}(G)$ is given by

$$
(\alpha, g)(\beta, h)=(\alpha \beta, g \alpha(h)) .
$$

Notice that $\operatorname{Hol}(G)$ acts on $G$ transitively under the following action:

$$
g^{(\alpha, h)}=\pi_{2}((\alpha, h)(1, g))=h \alpha(g)
$$

for all $\alpha \in \operatorname{Aut}(G)$ and $g, h \in G$, where $\pi_{2}: \operatorname{Hol}(G) \rightarrow G$ is the projection map given by $\pi_{2}((\alpha, g))=g$. It follows that the stabilizer of any element of $G$ in $\operatorname{Hol}(G)$ is isomorphic to $\operatorname{Aut}(G)$.

Let $\mathcal{G}$ be a regular subgroup of $\operatorname{Hol}(G)$. Then it is not difficult to see that for each $g \in G$, there exists a unique element $(\alpha, h) \in \mathcal{G}$ such that $g^{(\alpha, h)}=h \alpha(g)=1$. Let $\operatorname{Reg}(G)$ denote the set of all regular subgroups of $\operatorname{Hol}(G)$. Then $\operatorname{Hol}(G)$ acts on $\operatorname{Reg}(G)$ by conjugation. With this setting, we have the following easy observation, which plays a key role in what follows.

Lemma 2.1. Aut $(G)$, as a subgroup of $\operatorname{Hol}(G)$, is action-closed with respect to the conjugation action of $\operatorname{Hol}(G)$ on $\operatorname{Reg}(G)$.

Proof. Let $\mathcal{G} \in \operatorname{Reg}(G)$ and $(\alpha, h) \in \operatorname{Hol}(G)$. Then there exists an element $\left(\alpha_{1}, h_{1}\right) \in \mathcal{G}$ such that $h^{\left(\alpha_{1}, h_{1}\right)}=h_{1} \alpha_{1}(h)=1$. Notice that

$$
\left(\alpha_{1}, h_{1}\right)(\alpha, h)=\left(\alpha_{1} \alpha, h_{1} \alpha_{1}(h)\right)=\left(\alpha_{1} \alpha, 1\right) .
$$

Let $\beta:=\alpha_{1} \alpha$, which lies in $\operatorname{Aut}(G)$. Thus,

$$
\mathcal{G}^{(\beta, 1)}=\mathcal{G}^{\left(\alpha_{1}, h_{1}\right)(\alpha, h)}=\left(\mathcal{G}^{\left(\alpha_{1}, h_{1}\right)}\right)^{(\alpha, h)}=\mathcal{G}^{(\alpha, h)} .
$$

Proof is now complete. 
The preceding lemma enables us to get the following generalization of [15. Proposition 4.3].

Theorem 2.2. Let $(G,+)$ be a group. Then non-isomorphic skew left braces $(G,+, \circ)$ are in bijective correspondence with conjugacy classes of regular subgroups in $\operatorname{Hol}(G,+)$. Moreover, if $G$ is a p-group for some prime $p$, then non-isomorphic skew left brace structures over $G$ are in bijective correspondence with $\operatorname{Aut}(G)$ - conjugacy classes of regular subgroups of any Sylow p-subgroup of $\operatorname{Hol}(G,+)$.

Proof. The first assertion follows from [15, Proposition 4.3] along with Lemma 2.1. Let $\mathcal{S}$ be a fixed Sylow $p$-subgroup of $\operatorname{Hol}(G,+)$ and $\mathcal{S}^{\prime}$ any other Sylow $p$-subgroup of $\operatorname{Hol}(G,+)$. In the light of first assertion, we only need to observe that any regular subgroup of $\mathcal{S}^{\prime}$ lies in the $\operatorname{Hol}(G,+)$ - conjugacy class of some regular subgroup of $\mathcal{S}$. But this is obvious by Sylow theory.

As a result, we get the following algorithm which improves [15, Algorithm 5.1].

Algorithm 2.3. For a finite group $(G,+)$, the following sequence of computations constructs all non-isomorphic skew left braces $(G,+, \circ)$ :

(1) Compute $\operatorname{Hol}(G,+)$.

(2) Compute the list of regular subgroups of $\operatorname{Hol}(G)$ of order $|G|$ up to conjugation.

(3) For each representative $\mathcal{G}$ of regular subgroups of $\operatorname{Hol}(G)$, construct the map $\chi: G \rightarrow$ $\mathcal{G}$ given by $g \mapsto\left(f, f(g)^{-1}\right)$, where $\left(f, f(g)^{-1}\right) \in \mathcal{G}$. The triple $(\mathcal{G}, G, \chi)$ yields a skew left brace $(G,+, \circ)$ with $\circ: G \times G \rightarrow G$ given by $g_{1} \circ g_{2}=\chi^{-1}\left(\chi\left(g_{1}\right) \chi\left(g_{2}\right)\right)$ for all $g_{1}, g_{2} \in G$.

As remarked in [15] too, for enumerating skew left braces with additive group $(G,+)$ we only need first two steps of this algorithm.

We also have the following algorithm for finite $p$-groups.

Algorithm 2.4. For a finite p-group $(G,+)$, the following sequence of computations constructs all non-isomorphic skew left braces $(G,+, \circ)$ :

(1) Compute $\operatorname{Hol}(G,+)$.

(2) Compute a representative $\mathcal{S}$ of the conjugacy class of Sylow p-subgroups of $\operatorname{Hol}(G,+)$.

(3) Compute the list of regular subgroups of $\mathcal{S}$ of order $|G|$ up to conjugation by the elements of $\operatorname{Aut}(G)$.

(4) For each representative $\mathcal{G}$ of regular subgroups of $\mathcal{S}$ under conjugation action of $\operatorname{Aut}(G)$, construct the map $\chi: G \rightarrow \mathcal{G}$ given by $g \mapsto\left(f, f(g)^{-1}\right)$, where $\left(f, f(g)^{-1}\right) \in$ $\mathcal{G}$. The triple $(\mathcal{G}, G, \chi)$ yields a skew left brace $(G,+, \circ)$ with $\circ: G \times G \rightarrow G$ given by $g_{1} \circ g_{2}=\chi^{-1}\left(\chi\left(g_{1}\right) \chi\left(g_{2}\right)\right)$ for all $g_{1}, g_{2} \in G$.

Notice that for enumerating skew left braces with finite additive $p$-group $(G,+)$ we need first three steps of this algorithm. We conclude this section by reproducing the proof of the following fact.

Proposition 2.5. Let $\mathcal{S}$ be a Sylow p-subgroup of $\operatorname{Hol}(G)$ of a finite p-group $G$. Then the union of $\operatorname{Aut}(G)$ - conjugacy classes of the regular subgroups of $\mathcal{S}$ constitutes the set of all regular subgroups of $\operatorname{Hol}(G)$.

Proof. Let $\mathcal{G}$ be an arbitrary regular subgroup of $\operatorname{Hol}(G)$. Then $\mathcal{G}$, being of order $|G|$, is a subgroup of some Sylow $p$-subgroup $\mathcal{S}^{\prime}$ of $\operatorname{Hol}(G)$. By Sylow theory, we know that there exists an element $(\phi, y) \in \operatorname{Hol}(G)$ such that $\mathcal{S}=\left(\mathcal{S}^{\prime}\right)^{(\phi, y)}$. Thus $\mathcal{G}^{(\phi, y)}$ is a subgroup of $\mathcal{S}$. It follows from the proof of Lemma 2.1 that $\mathcal{G}^{(\phi, y)}=\mathcal{G}^{(\beta, 1)}$ for some $\beta \in \operatorname{Aut}(G)$. A routine calculation now shows that $\mathcal{G}^{(\beta, 1)}$ is a regular subgroup of $\mathcal{S}$. Indeed, if $x^{\left((\psi, z)^{(\beta, 1)}\right)}=x$ for some $(\psi, z) \in \mathcal{G}$ and $x \in G$, then it follows that $\beta(x)^{(\psi, z)}=\beta(x)$, which is not possible. This proves that the action of $\mathcal{G}^{(\beta, 1)}$ is free on $G$. That the action is transitive, is left as an easy exercise, and the proof is complete. 
We remark that on the lines of proof of the preceding proposition, we can easily show that for an arbitrary finite group $G, \operatorname{Hol}(G)$ acts on $\operatorname{Reg}(G)$ by conjugation. We have used this fact above without proof as it is well known.

\section{Computations}

Throughout this section, for a given positive integer $n, b(n)$ and $s(n)$, respectively, denote the total number of left braces and skew left braces of order $n$. For each such $n, p f(n)$ stands for the prime factorization of $n$. Computations in this section are carried out using Algorithm 2.3. The following table remedies some gaps in the list obtained in 22 .

\begin{tabular}{|c|cccccccc|}
\hline$n$ & 32 & 54 & 64 & 72 & 80 & 81 & 96 & 108 \\
$b(n)$ & 25281 & 80 & $?$ & 489 & 1985 & 804 & 195971 & 494 \\
$s(n)$ & 1223061 & 1028 & $?$ & 17790 & 74120 & 8436 & $?$ & 11223 \\
\hline$n$ & 112 & 120 & 126 & 128 & 136 & 144 & 147 & 150 \\
$b(n)$ & 1671 & 395 & 36 & $?$ & 108 & 10215 & 9 & 19 \\
$s(n)$ & 65485 & 22711 & 990 & $?$ & 986 & 3013486 & 123 & 401 \\
\hline$n$ & 152 & 158 & 160 & 162 & 164 & 165 & 166 & 168 \\
$b(n)$ & 90 & 2 & 209513 & 1374 & 11 & 2 & 2 & 443 \\
$s(n)$ & 800 & 6 & $?$ & 45472 & 43 & 12 & 6 & 28505 \\
\hline
\end{tabular}

TABLE 1. Some missing values from [22]

We now enumerate $b(n)$ and $s(n)$ for $n \leq 868$ except some cases for which computations are too big to be handled by our computer. We have given a lower bound on the number of skew left braces of order $3^{5}$, by taking into account the additive groups with Group Id's $[243, m]$, where $m=1, \ldots, 31,33,37,38,40,48, \ldots, 63,65,66,67$. By the Group Id we mean the group identification of a group of given order in The Small Groups Library 12 implemented in GAP and MAGMA. 


\begin{tabular}{|c|c|c|c|c|c|c|c|c|c|c|}
\hline$n$ & 169 & 170 & 171 & 172 & 173 & 174 & 175 & 176 & 177 & 178 \\
\hline$b(n)$ & 4 & 4 & 14 & 9 & 1 & 4 & 4 & 1670 & 1 & 2 \\
\hline$s(n)$ & 4 & 36 & 80 & 29 & 1 & 36 & 4 & 65466 & 1 & 6 \\
\hline$n$ & 179 & 180 & 181 & 182 & 183 & 184 & 185 & 186 & 187 & 188 \\
\hline$b(n)$ & 1 & 129 & 1 & 4 & 2 & 90 & 1 & 6 & 1 & 9 \\
\hline$s(n)$ & 1 & 5849 & 1 & 36 & 8 & 800 & 1 & 78 & 1 & 29 \\
\hline$n$ & 189 & 190 & 191 & 192 & 193 & 194 & 195 & 196 & 197 & 198 \\
\hline$b(n)$ & 165 & 4 & 1 & $?$ & 1 & 2 & 2 & 41 & 1 & 16 \\
\hline$s(n)$ & 4560 & 36 & 1 & $?$ & 1 & 6 & 8 & 389 & 1 & 294 \\
\hline$n$ & $\begin{array}{l}199 \\
\end{array}$ & 200 & 201 & 202 & 203 & 204 & 205 & 206 & 207 & 208 \\
\hline$b(n)$ & 1 & 568 & 2 & 2 & 2 & 28 & 2 & 2 & 4 & 1984 \\
\hline$s(n)$ & 1 & 23471 & 8 & 6 & 16 & 410 & 12 & 6 & 4 & 74104 \\
\hline$n$ & 209 & 210 & 211 & 212 & 213 & 214 & 215 & 216 & 217 & 218 \\
\hline$b(n)$ & 1 & 12 & 1 & 11 & 1 & 2 & 1 & 5308 & 1 & 2 \\
\hline$s(n)$ & 1 & 468 & 1 & 43 & 1 & 6 & 1 & 523768 & 1 & 6 \\
\hline$n$ & 219 & 220 & 221 & 222 & 223 & 224 & 225 & 226 & 227 & 228 \\
\hline$b(n)$ & 2 & 36 & 1 & 6 & 1 & 195483 & 21 & 2 & 1 & 34 \\
\hline$s(n)$ & 8 & 702 & 1 & 78 & 1 & $?$ & 61 & 6 & 1 & 606 \\
\hline $\bar{n}$ & 229 & 230 & 231 & 232 & 233 & 234 & 235 & 256 & 237 & 238 \\
\hline$b(n)$ & 1 & 4 & 2 & 106 & 1 & 36 & 1 & 9 & 2 & 4 \\
\hline$s(n)$ & 1 & 36 & 8 & 944 & 1 & 990 & 1 & 29 & 8 & 36 \\
\hline$n$ & 239 & 240 & 241 & 242 & 243 & 244 & 245 & 246 & 247 & 248 \\
\hline$b(n)$ & 1 & 10518 & 1 & 8 & 598065 & 11 & 4 & 4 & 1 & 90 \\
\hline$s(n)$ & 1 & 4642485 & 1 & 57 & $>27447027$ & 43 & 4 & 36 & 1 & 800 \\
\hline$n$ & 249 & 250 & 251 & 252 & 253 & 254 & 255 & 256 & 257 & 258 \\
\hline$b(n)$ & 1 & 104 & 1 & 229 & 2 & 2 & 1 & $?$ & 1 & 6 \\
\hline$s(n)$ & 1 & 1492 & 1 & 11541 & 24 & 6 & 1 & $?$ & 1 & 78 \\
\hline$n$ & 259 & 260 & 261 & 262 & 263 & 264 & 265 & 266 & 267 & 268 \\
\hline$b(n)$ & 1 & 35 & 4 & 2 & 1 & 345 & 1 & 4 & 1 & 9 \\
\hline$s(n)$ & 1 & 739 & 4 & 6 & 1 & 20231 & 1 & 36 & 1 & 29 \\
\hline$n$ & 269 & 270 & 271 & 272 & 273 & 274 & 275 & 276 & 277 & 278 \\
\hline$b(n)$ & 1 & 160 & 1 & 2014 & 5 & 2 & 13 & 24 & 1 & 2 \\
\hline$s(n)$ & 1 & 6168 & 1 & 74960 & 113 & 6 & 93 & 324 & 1 & 6 \\
\hline$n$ & 279 & 280 & 281 & 282 & 283 & 284 & 285 & 286 & 287 & 288 \\
\hline$b(n)$ & 11 & 385 & 1 & 4 & 1 & 9 & 2 & 4 & 1 & 1392959 \\
\hline$s(n)$ & 47 & 22295 & 1 & 36 & 1 & 29 & 8 & 36 & 1 & $?$ \\
\hline$n$ & 289 & 290 & 291 & 292 & 293 & 294 & 295 & 296 & 297 & 298 \\
\hline$b(n)$ & 4 & 4 & 2 & 11 & 1 & 31 & 1 & 106 & 37 & 2 \\
\hline$s(n)$ & 4 & 36 & 8 & 43 & 1 & 2152 & 1 & 944 & 101 & 6 \\
\hline$n$ & 299 & 300 & 301 & 302 & 303 & 304 & 305 & 306 & 307 & 308 \\
\hline$b(n)$ & 1 & 152 & 2 & 2 & 1 & 1670 & 2 & 16 & 1 & 23 \\
\hline$s(n)$ & 1 & 8222 & 16 & 6 & 1 & 65466 & 12 & 294 & 1 & 311 \\
\hline$n$ & 309 & 310 & 311 & 312 & 313 & 314 & 315 & 316 & 317 & 318 \\
\hline$b(n)$ & 2 & 6 & 1 & 507 & 1 & 2 & 11 & 9 & 1 & 4 \\
\hline$s(n)$ & 8 & 94 & 1 & 32075 & 1 & 6 & 47 & 29 & 1 & 36 \\
\hline $\bar{n}$ & 319 & 320 & 321 & 322 & 323 & 324 & 325 & 326 & 327 & 328 \\
\hline$b(n)$ & 1 & $?$ & 1 & 4 & 1 & 10225 & 4 & 2 & 2 & 108 \\
\hline$s(n)$ & 1 & $?$ & 1 & 36 & 1 & $?$ & 4 & 6 & 8 & 986 \\
\hline$n$ & 329 & 330 & 331 & 332 & 333 & 334 & 335 & 336 & 337 & 338 \\
\hline$b(n)$ & 1 & 12 & 1 & 9 & 14 & 2 & 1 & 10990 & 1 & 8 \\
\hline$s(n)$ & 1 & 564 & 1 & 29 & 80 & 6 & 1 & 5247711 & 1 & 59 \\
\hline$n$ & 339 & 340 & 341 & 342 & 343 & 344 & 345 & 346 & 347 & 348 \\
\hline$b(n)$ & 1 & 35 & 1 & 42 & 61 & 90 & 1 & 2 & 1 & 28 \\
\hline$s(n)$ & 1 & 739 & 1 & 1164 & 373 & 800 & 1 & 6 & 1 & 410 \\
\hline
\end{tabular}

TABle 2. Further Computations 


\begin{tabular}{|c|c|c|c|c|c|c|c|c|c|c|}
\hline$n$ & 349 & 350 & 351 & 352 & 353 & 354 & 355 & 356 & 357 & 358 \\
\hline$b(n)$ & 1 & 16 & 166 & 195479 & 1 & 4 & 2 & 11 & 2 & 2 \\
\hline$s(n)$ & 1 & 306 & 4591 & $?$ & 1 & 36 & 12 & 43 & 8 & 6 \\
\hline$n$ & 359 & 360 & 361 & 362 & 363 & 364 & 365 & 366 & 367 & 368 \\
\hline$b(n)$ & 1 & 2035 & 4 & 2 & 5 & 27 & 1 & 6 & 1 & 1670 \\
\hline$s(n)$ & 1 & 535713 & 4 & 6 & 20 & 395 & 1 & 78 & 1 & 65466 \\
\hline$n$ & 369 & 370 & $\overline{371}$ & 372 & 373 & 374 & 375 & 376 & 377 & 378 \\
\hline$b(n)$ & 4 & 4 & 1 & 34 & 1 & 4 & 54 & 90 & 1 & 548 \\
\hline$s(n)$ & 4 & 36 & 1 & 606 & 1 & 36 & 253 & 800 & 1 & 47244 \\
\hline$n$ & 379 & 380 & 381 & 382 & 383 & 384 & 385 & 386 & 387 & 388 \\
\hline$b(n)$ & 1 & 27 & 2 & 2 & 1 & $?$ & 2 & 2 & 11 & 11 \\
\hline$s(n)$ & 1 & 395 & 8 & 6 & 1 & $?$ & 12 & 6 & 47 & 43 \\
\hline$n$ & 389 & 390 & 391 & 392 & 393 & 394 & 395 & 396 & 397 & 398 \\
\hline$b(n)$ & 1 & 12 & 1 & 463 & 1 & 2 & 1 & 111 & 1 & 2 \\
\hline$s(n)$ & 1 & 468 & 1 & 18078 & 1 & 6 & 1 & 4985 & 1 & 6 \\
\hline$n$ & 399 & 400 & 401 & 402 & 403 & 404 & 405 & 406 & 407 & 408 \\
\hline$b(n)$ & 5 & 12744 & 1 & 6 & 1 & 11 & 805 & 6 & 1 & 399 \\
\hline$s(n)$ & 113 & 3618636 & 1 & 78 & 1 & 43 & 8453 & 110 & 1 & 22923 \\
\hline$n$ & 409 & 410 & $\overline{411}$ & 412 & 413 & $\overline{414}$ & 415 & 416 & 417 & 418 \\
\hline$b(n)$ & 1 & 6 & 1 & 9 & 1 & 16 & 1 & 209507 & 2 & 4 \\
\hline$s(n)$ & 1 & 94 & 1 & 29 & 1 & 294 & 1 & $?$ & 8 & 36 \\
\hline$n$ & 419 & 420 & $\overline{421}$ & 422 & 423 & 424 & 425 & 426 & 427 & $\overline{428}$ \\
\hline$b(n)$ & 1 & 104 & 1 & 2 & 4 & 106 & 4 & 4 & 1 & 9 \\
\hline$s(n)$ & 1 & 9052 & 1 & 6 & 4 & 944 & 4 & 36 & 1 & 29 \\
\hline$n$ & 429 & 430 & 431 & 432 & 433 & 434 & 435 & 436 & 437 & 438 \\
\hline$b(n)$ & 2 & 4 & 1 & 115708 & 1 & 4 & 1 & 11 & 1 & 6 \\
\hline$s(n)$ & 8 & 36 & 1 & $?$ & 1 & 36 & 1 & 43 & 1 & 78 \\
\hline$n$ & 439 & 440 & $\overline{441}$ & 442 & 443 & 444 & 445 & 446 & 447 & 448 \\
\hline$b(n)$ & 1 & 474 & 55 & 4 & 1 & 40 & 1 & 2 & 1 & $?$ \\
\hline$s(n)$ & 1 & 31970 & 1110 & 36 & 1 & 782 & 1 & 6 & 1 & $?$ \\
\hline$n$ & 449 & 450 & 451 & 452 & 453 & 454 & 455 & 456 & 457 & 458 \\
\hline$b(n)$ & 1 & 82 & 1 & 11 & 2 & 2 & 1 & 441 & 1 & 2 \\
\hline$s(n)$ & 1 & 3797 & 1 & 43 & 8 & 6 & 1 & 28447 & 1 & 6 \\
\hline$n$ & 459 & 460 & $\overline{461}$ & 462 & 463 & 464 & 465 & 466 & 467 & 468 \\
\hline$b(n)$ & 37 & 27 & 1 & 12 & 1 & 1984 & 4 & 2 & 1 & 267 \\
\hline$s(n)$ & 101 & 395 & 1 & 468 & 1 & 74104 & 66 & 6 & 1 & 13941 \\
\hline$n$ & 469 & 470 & 471 & 472 & 473 & 474 & 475 & 476 & 477 & 478 \\
\hline$b(n)$ & 1 & 4 & 2 & 90 & 1 & 6 & 4 & 27 & 4 & 2 \\
\hline$s(n)$ & 1 & 36 & 8 & 800 & 1 & 78 & 4 & 395 & 4 & 6 \\
\hline$n$ & 479 & 480 & 481 & 482 & 483 & 484 & 485 & 486 & 487 & 488 \\
\hline$b(n)$ & 1 & $?$ & 1 & 2 & 2 & 41 & 1 & 639775 & 1 & 106 \\
\hline$s(n)$ & 1 & $?$ & 1 & 6 & 8 & 421 & 1 & $?$ & 1 & 944 \\
\hline$n$ & 489 & 490 & 491 & 492 & 493 & 494 & 495 & 496 & 497 & 498 \\
\hline$b(n)$ & 2 & 16 & 1 & 28 & 1 & 4 & 8 & 1670 & 2 & 4 \\
\hline$s(n)$ & 8 & 318 & 1 & 410 & 1 & 36 & 48 & 65466 & 16 & 36 \\
\hline$n$ & 499 & 500 & 501 & 502 & 503 & 504 & 505 & 506 & 507 & 508 \\
\hline$b(n)$ & 1 & 634 & 1 & 2 & 1 & 3249 & 2 & 6 & 9 & 9 \\
\hline$s(n)$ & 1 & 21252 & 1 & 6 & 1 & 871013 & 12 & 142 & 135 & 29 \\
\hline$n$ & 509 & 510 & 511 & 512 & 513 & 514 & 515 & 516 & 517 & 518 \\
\hline$b(n)$ & 1 & 8 & 1 & $?$ & 189 & 2 & 1 & 34 & 1 & 4 \\
\hline$s(n)$ & 1 & 216 & 1 & $?$ & 5055 & 6 & 1 & 606 & 1 & 36 \\
\hline$n$ & 519 & 520 & 521 & 522 & 523 & 524 & 525 & 526 & 527 & 528 \\
\hline$b(n)$ & 1 & 484 & 1 & 16 & 1 & 9 & 10 & 2 & 1 & 9274 \\
\hline$s(n)$ & 1 & 28714 & 1 & 294 & 1 & 29 & 112 & 6 & 1 & 4381956 \\
\hline
\end{tabular}

TABLE 3. Further Computations 


\begin{tabular}{|c|c|c|c|c|c|c|c|c|c|c|}
\hline$n$ & 529 & 530 & 531 & 532 & 533 & 534 & 535 & 536 & 537 & 538 \\
\hline$b(n)$ & 4 & 4 & 4 & 23 & 1 & 4 & 1 & 90 & 1 & 2 \\
\hline$s(n)$ & 4 & 36 & 4 & 311 & 1 & 36 & 1 & 800 & 1 & 6 \\
\hline$n$ & 539 & 540 & 541 & 542 & 543 & 544 & 545 & 546 & 547 & 548 \\
\hline$b(n)$ & 4 & 1342 & 1 & 2 & 2 & 210043 & 1 & 24 & 1 & 11 \\
\hline$s(n)$ & 4 & 148151 & 1 & 6 & 8 & $?$ & 1 & 2664 & 1 & 43 \\
\hline$n$ & 549 & 550 & 551 & 552 & 553 & 554 & 555 & 556 & 557 & 558 \\
\hline$b(n)$ & 11 & 40 & 1 & 345 & 1 & 2 & 2 & 9 & 1 & 36 \\
\hline$s(n)$ & 47 & 1370 & 1 & 20231 & 1 & 6 & 8 & 29 & 1 & 990 \\
\hline$n$ & 559 & 560 & 561 & 562 & 563 & 564 & 565 & 566 & 567 & 568 \\
\hline$b(n)$ & 1 & 10423 & 1 & 2 & 1 & 24 & 1 & 2 & 7196 & 90 \\
\hline$s(n)$ & 1 & 4633376 & 1 & 6 & 1 & 324 & 1 & 6 & 2253564 & 800 \\
\hline$n$ & 569 & 570 & 571 & 572 & 573 & 574 & 575 & 576 & 577 & 578 \\
\hline$b(n)$ & 1 & 12 & 1 & 27 & 1 & 4 & 4 & $?$ & 1 & 8 \\
\hline$s(n)$ & 1 & 468 & 1 & 395 & 1 & 36 & 4 & $?$ & 1 & 63 \\
\hline$n$ & 579 & 580 & 581 & 582 & 583 & 584 & 585 & 586 & 587 & 588 \\
\hline$b(n)$ & 2 & 35 & 1 & 6 & 1 & 108 & 11 & 2 & 1 & 202 \\
\hline$s(n)$ & 8 & 739 & 1 & 78 & 1 & 986 & 47 & 6 & 1 & 21836 \\
\hline$n$ & 589 & 590 & 591 & 592 & 593 & 594 & 595 & 596 & 597 & 598 \\
\hline$b(n)$ & 1 & 4 & 1 & 1984 & 1 & 160 & 1 & 11 & 2 & 4 \\
\hline$s(n)$ & 1 & 36 & 1 & 74104 & 1 & 6168 & 1 & 43 & 8 & 36 \\
\hline$n$ & 599 & 600 & 601 & 602 & 603 & 604 & 605 & 606 & 607 & 608 \\
\hline$b(n)$ & 1 & 2413 & 1 & 6 & 11 & 9 & 10 & 4 & 1 & 195479 \\
\hline$s(n)$ & 1 & 659897 & 1 & 110 & 47 & 29 & 409 & 36 & 1 & $?$ \\
\hline$n$ & 609 & 610 & 611 & 612 & 613 & 614 & 615 & 616 & 617 & 618 \\
\hline$b(n)$ & 3 & 6 & 1 & 129 & 1 & 2 & 2 & 335 & 1 & 6 \\
\hline$s(n)$ & 25 & 94 & 1 & 5835 & 1 & 6 & 12 & 19885 & 1 & 78 \\
\hline$n$ & 619 & 620 & 621 & 622 & 623 & 624 & 625 & 626 & 627 & 628 \\
\hline$b(n)$ & 1 & 36 & 37 & 2 & 1 & 12547 & 2308 & 2 & 2 & 11 \\
\hline$s(n)$ & 1 & 702 & 101 & 6 & 1 & 5595183 & 69032 & 6 & 8 & 43 \\
\hline$n$ & 629 & 630 & 631 & 632 & 633 & 634 & 635 & 636 & 637 & 638 \\
\hline$b(n)$ & 1 & 72 & 1 & 90 & 2 & 2 & 1 & 28 & 4 & 4 \\
\hline$s(n)$ & 1 & 5940 & 1 & 800 & 8 & 6 & 1 & 410 & 4 & 36 \\
\hline$n$ & 639 & 640 & 641 & 642 & 643 & 644 & 645 & 646 & 647 & 648 \\
\hline$b(n)$ & 4 & $?$ & 1 & 4 & 1 & 23 & 2 & 4 & 1 & 91071 \\
\hline$s(n)$ & 4 & $?$ & 1 & 36 & 1 & 311 & 8 & 36 & 1 & $?$ \\
\hline$n$ & 649 & 650 & 651 & 652 & 653 & 654 & 655 & 656 & 657 & 658 \\
\hline$b(n)$ & 1 & 16 & 5 & 9 & 1 & 6 & 2 & 2010 & 14 & 4 \\
\hline$s(n)$ & 1 & 306 & 113 & 29 & 1 & 78 & 12 & 74860 & 80 & 36 \\
\hline$n$ & 659 & 660 & 661 & 662 & 663 & 664 & 665 & 666 & 667 & 668 \\
\hline$b(n)$ & 1 & 100 & 1 & 2 & 2 & 90 & 1 & 42 & 1 & 9 \\
\hline$s(n)$ & 1 & 9346 & 1 & 6 & 8 & 800 & 1 & 1164 & 1 & 29 \\
\hline$n$ & 669 & 670 & 671 & 672 & 673 & 674 & 675 & 676 & 677 & 678 \\
\hline$b(n)$ & 2 & 4 & 1 & $?$ & 1 & 2 & 232 & 51 & 1 & 4 \\
\hline$s(n)$ & 8 & 36 & 1 & $?$ & 1 & 6 & 3682 & 791 & 1 & 36 \\
\hline$n$ & 679 & 680 & 681 & 682 & 683 & 684 & 685 & 686 & 687 & 688 \\
\hline$b(n)$ & 1 & 492 & 1 & 4 & 1 & 259 & 1 & 128 & 2 & 1670 \\
\hline$s(n)$ & 1 & 29698 & 1 & 36 & 1 & 12723 & 1 & 2084 & 8 & 65466 \\
\hline$n$ & 689 & 690 & 691 & 692 & 693 & 694 & 695 & 696 & 697 & 698 \\
\hline$b(n)$ & 2 & 8 & 1 & 11 & 11 & 2 & 1 & 395 & 1 & 2 \\
\hline$s(n)$ & 28 & 216 & 1 & 43 & 47 & 6 & 1 & 22667 & 1 & 6 \\
\hline$n$ & 699 & 700 & 701 & 702 & 7703 & 704 & 705 & 706 & 707 & 708 \\
\hline$b(n)$ & 1 & 126 & 1 & 550 & 1 & $?$ & 1 & 2 & 1 & 24 \\
\hline$s(n)$ & 1 & 7102 & 1 & 47374 & 1 & $?$ & 1 & 6 & 1 & 324 \\
\hline
\end{tabular}

TABLE 4. Further Computations 


\begin{tabular}{|c|c|c|c|c|c|c|c|c|c|c|}
\hline$n$ & 709 & 710 & 711 & 712 & 713 & 714 & 715 & 716 & 717 & 718 \\
\hline$b(n)$ & 1 & 6 & 11 & 108 & 1 & 12 & 2 & 9 & 1 & 2 \\
\hline$s(n)$ & 1 & 94 & 47 & 986 & 1 & 468 & 12 & 29 & 1 & 6 \\
\hline$n$ & 719 & 720 & 721 & 722 & 723 & 724 & 725 & 726 & 727 & 728 \\
\hline$b(n)$ & 1 & 65074 & 1 & 8 & 2 & 11 & 4 & 19 & 1 & 385 \\
\hline$s(n)$ & 1 & $?$ & 1 & 65 & 8 & 43 & 4 & 466 & 1 & 22295 \\
\hline$n$ & 729 & 730 & 731 & 732 & 733 & 734 & 735 & 736 & 737 & 738 \\
\hline$b(n)$ & $?$ & 4 & 1 & 40 & 1 & 2 & 9 & 195479 & 2 & 16 \\
\hline$s(n)$ & $?$ & 36 & 1 & 782 & 1 & 6 & 123 & $?$ & 24 & 294 \\
\hline$n$ & 739 & 740 & 741 & 742 & 743 & 744 & 745 & 746 & 747 & 748 \\
\hline$b(n)$ & 1 & 35 & 5 & 4 & 1 & 441 & 1 & 2 & 4 & 27 \\
\hline$s(n)$ & 1 & 739 & 113 & 36 & 1 & 28447 & 1 & 6 & 4 & 395 \\
\hline$n$ & 749 & 750 & 751 & 752 & 753 & 754 & 755 & 756 & 757 & 758 \\
\hline$b(n)$ & 1 & 224 & 1 & 1670 & 1 & 4 & 2 & 3757 & 1 & 2 \\
\hline$s(n)$ & 1 & 10001 & 1 & 65466 & 1 & 36 & 12 & 794193 & 1 & 6 \\
\hline$n$ & 759 & 760 & 761 & 762 & 763 & 764 & 765 & 766 & 767 & 768 \\
\hline$b(n)$ & 2 & 384 & 1 & 6 & 1 & 9 & 4 & 2 & 1 & $?$ \\
\hline$s(n)$ & 24 & 22278 & 1 & 78 & 1 & 29 & 4 & 6 & 1 & $?$ \\
\hline$n$ & 769 & 770 & 771 & 772 & 773 & 774 & 775 & 776 & 777 & 778 \\
\hline$b(n)$ & 1 & 12 & 1 & 11 & 1 & 36 & 13 & 108 & 5 & 2 \\
\hline$s(n)$ & 1 & 564 & 1 & 43 & 1 & 990 & 93 & 986 & 113 & 6 \\
\hline$n$ & 779 & 780 & 781 & 782 & 783 & 784 & 785 & 786 & 787 & 788 \\
\hline$b(n)$ & 1 & 128 & 1 & 4 & 37 & 9998 & 1 & 4 & 1 & 11 \\
\hline$s(n)$ & 1 & 13320 & 1 & 36 & 101 & 3074483 & 1 & 36 & 1 & 43 \\
\hline$n$ & 789 & 790 & 791 & 792 & 793 & 794 & 795 & 796 & 797 & 798 \\
\hline$b(n)$ & 1 & 4 & 2 & 1771 & 1 & 2 & 1 & 9 & 1 & 24 \\
\hline$s(n)$ & 1 & 36 & 16 & 484183 & 1 & 6 & 1 & 29 & 1 & 2664 \\
\hline$n$ & 799 & 800 & 801 & 802 & 803 & 804 & 805 & 806 & 807 & 808 \\
\hline$b(n)$ & 1 & $?$ & 4 & 2 & 1 & 34 & 1 & 4 & 1 & 106 \\
\hline$s(n)$ & 1 & $?$ & 4 & 6 & 1 & 606 & 1 & 36 & 1 & 944 \\
\hline$n$ & 809 & 810 & 811 & 812 & 813 & 814 & 815 & 816 & 817 & 818 \\
\hline$b(n)$ & 1 & 2751 & 1 & 38 & 2 & 4 & 1 & 10604 & 1 & 2 \\
\hline$s(n)$ & 1 & 272960 & 1 & 920 & 8 & 36 & 1 & 4658179 & 1 & 6 \\
\hline$n$ & 819 & 820 & 821 & 822 & 823 & 824 & 825 & 826 & 827 & 828 \\
\hline$b(n)$ & 41 & 46 & 1 & 4 & 1 & 90 & 14 & 4 & 1 & 111 \\
\hline$s(n)$ & 1337 & 1212 & 1 & 36 & 1 & 800 & 105 & 36 & 1 & 4985 \\
\hline$n$ & 829 & 830 & 831 & 832 & 833 & 834 & 835 & 836 & 837 & 838 \\
\hline$b(n)$ & 1 & 4 & 2 & $?$ & 4 & 6 & 1 & 23 & 165 & 2 \\
\hline$s(n)$ & 1 & 36 & 8 & $?$ & 4 & 78 & 1 & 311 & 4560 & 6 \\
\hline$n$ & 839 & 840 & 841 & 842 & 843 & 844 & 845 & 846 & 847 & 848 \\
\hline$b(n)$ & 1 & 1933 & 4 & 2 & 1 & 9 & 4 & 16 & 4 & 1984 \\
\hline$s(n)$ & 1 & 878779 & 4 & 6 & 1 & 27 & 4 & 294 & 4 & 74104 \\
\hline$n$ & 849 & 850 & 851 & 852 & 853 & 854 & 855 & 856 & 857 & 858 \\
\hline$b(n)$ & 2 & 16 & 1 & 24 & 1 & 4 & 14 & 90 & 1 & 12 \\
\hline$s(n)$ & 8 & 306 & 1 & 324 & 1 & 36 & 80 & 800 & 1 & 468 \\
\hline$n$ & 859 & 860 & 861 & 862 & 863 & 864 & 865 & 866 & 867 & 868 \\
\hline$b(n)$ & 1 & 27 & 2 & 2 & 1 & $?$ & 1 & 2 & 5 & 23 \\
\hline$s(n)$ & 1 & 395 & 8 & 6 & 1 & $?$ & 1 & 6 & 26 & 311 \\
\hline
\end{tabular}

TABLE 5. Further Computations

We now record some partial computations considering specific additive groups of given orders. 


\begin{tabular}{|c|c|c|c|c|c|c|}
\hline Group Id & {$[64,1]$} & {$[64,2]$} & {$[64,26]$} & {$[64,50]$} & {$[64,55]$} & {$[64,83]$} \\
Number & 10 & 11354 & 2742 & 142 & $?$ & 734410 \\
\hline \hline Group Id & {$[64,183]$} & {$[64,192]$} & {$[64,246]$} & {$[64,260]$} & {$[64,267]$} & \\
Number & 3124 & $?$ & 253350 & 2189661 & 58558 & \\
\hline
\end{tabular}

TABLE 6. Enumerations of left braces of order 64

\begin{tabular}{|c|c|c|c|c|c|c|c|}
\hline Group Id & {$[480,4]$} & {$[480,199]$} & {$[480,212]$} & {$[480,919]$} & {$[480,934]$} & {$[480,1180]$} & {$[480,1213]$} \\
Number & 128 & $?$ & 4928 & 958965 & 99970 & $?$ & 39650 \\
\hline
\end{tabular}

TABLE 7. Enumerations of left braces of order 480

\section{Conclusion and Conjectures}

We start by presenting a comparison on the time taken ( in seconds) by 15, Algorithm 5.1] and Algorithm 2.3 for enumerating skew left braces of order 32 for select additive groups which took considerable amount of time on MAGMA.

\begin{tabular}{|c|c|c|c|c|c|c|}
\hline Group Id of the additive group & {$[32,23]$} & {$[32,24]$} & {$[32,25]$} & {$[32,28]$} & {$[32,29]$} & {$[32,30]$} \\
Number of skew brace structures & 39488 & 70400 & 138336 & 138336 & 138336 & 137526 \\
Time on Algorithm $5.1[13]$ & 11238 & 9808 & 18720 & 10193 & 10083 & 34005 \\
Time on Algorithm 2.3 & 539 & 709 & 1905 & 4308 & 3135 & 4658 \\
\hline \hline Group Id of the additive group & {$[32,31]$} & {$[32,32]$} & {$[32,33]$} & {$[32,45]$} & {$[32,47]$} & {$[32,51]$} \\
Number of skew brace structures & 70944 & 69236 & 91008 & 8015 & 7870 & 744 \\
Time on Algorithm $5.1[13]$ & 14568 & 18342 & 17222 & 130942 & 28848 & $\#$ \\
Time on Algorithm 2.3 & 4797 & 9302 & 8869 & 30 & 68 & 8 \\
\hline
\end{tabular}

TABLE 8. Time comparison on skew left braces of order 32

\# Program was stopped after running more than a month without result.

The data obtained above reveals that Algorithm 2.3 is very expensive, with respect to memory space and time, for handing the situation for prime power orders. So, one really needs to find a substitute for this algorithm. One may think of Algorithm 2.4 as a substitute. But unfortunately, it requires the conjugacy classes of regular subgroups of a given Sylow- $p$-subgroup (of the holomorph of a given finite $p$-group) to be computed in the whole holomorph, which is again very expensive. Although Algorithm 2.4 is not very efficient as such, we hope that it may be improved/modified to handle the computations on skew braces of prime power orders more efficiently.

We now present some conjectures suggested by the big data computed in above tables. It is known from [10] that for a prime integer $q \geq 5$,

$$
b(4 q)=\left\{\begin{array}{lll}
9, & \text { if } q \equiv 3 \quad \bmod 4 \\
11, & \text { if } q \equiv 1 \quad \bmod 4
\end{array}\right.
$$

and for prime integers $p$ and $q$ such that $q>p+1>3$,

$$
b\left(p^{2} q\right)= \begin{cases}4, & \text { if } 3 \nmid p-1 \\ p+8, & \text { if } 3 \mid p-1 \text { and } 9 \nmid p-1 \\ 2 p+8, & \text { if } 9 \mid p-1 .\end{cases}
$$

For skew left braces, we have 
Conjecture 4.1. Let $p$ and $q$ be prime integers. If $q \geq 5$, then

$$
s(4 q)= \begin{cases}29, & \text { if } q \equiv 3 \quad \bmod 4 \\ 43, & \text { if } q \equiv 1 \bmod 4\end{cases}
$$

and if $q>p+1>3$, then

$$
s\left(p^{2} q\right)= \begin{cases}4, & \text { if } p \nmid q-1 \\ 2 p^{2}+7 p+8, & \text { if } p \mid q-1 \text { and } p^{2} \nmid q-1 \\ 6 p^{2}+6 p+8, & \text { if } p^{2} \mid q-1 .\end{cases}
$$

For prime multiples of 8 and 12, we have

Conjecture 4.2. Let $p \geq 11$ be a prime integer. Then

$$
b(8 p)= \begin{cases}90, & \text { if } p \equiv 3,7 \bmod 8 \\ 106, & \text { if } p \equiv 5 \bmod 8 \\ 108, & \text { if } p \equiv 1 \bmod 8\end{cases}
$$

and

$$
s(8 p)= \begin{cases}800, & \text { if } p \equiv 3,7 \bmod 8 \\ 944, & \text { if } p \equiv 5 \bmod 8 \\ 986, & \text { if } p \equiv 1 \bmod 8\end{cases}
$$

Conjecture 4.3. Let $p \geq 7$ be a prime integer. Then

$$
b(12 p)=\left\{\begin{array}{lll}
24, & \text { if } p \equiv 11 \quad \bmod 12 \\
28, & \text { if } p \equiv 5 \quad \bmod 12 \\
34, & \text { if } p \equiv 7 \quad \bmod 12 \\
40, & \text { if } p \equiv 1 \quad \bmod 12 .
\end{array}\right.
$$

and

$$
s(12 p)= \begin{cases}324, & \text { if } p \equiv 11 \bmod 12 \\ 410, & \text { if } p \equiv 5 \bmod 12 \\ 606, & \text { if } p \equiv 7 \bmod 12 \\ 782, & \text { if } p \equiv 1 \quad \bmod 12 .\end{cases}
$$

Skew left braces of order $p q, p<q$ being prime integers, have been constructed very recently in [1, where it is shown that $s(p q)=1$ if $p \nmid q-1$ and $s(p q)=2 p+2$ otherwise. Going a step ahead, we have the following enumeration formula:

Conjecture 4.4. Let $p$ and $q$ be prime integers such that $q>p \geq 3$. Then

$$
b(2 p q)= \begin{cases}4, & \text { if } p \nmid q-1 \\ 6, & \text { if } p \mid q-1\end{cases}
$$

and

$$
s(2 p q)= \begin{cases}36, & \text { if } p \nmid q-1 \\ 8 p+54, & \text { if } p \mid q-1 .\end{cases}
$$

We close with the hope that the readers will be able to use the enormous data produced above to formulate many more conjectures according to their own need and interest. 
Acknowledgements. The third named author thanks L. Vendramin for supplying MAGMA codes for computing skew left braces and for his useful comments on the introduction, and acknowledges the support of DST-RSF Grant INT/RUS/RSF/P-2. The first and second named authors acknowledge the support from the RFBR-18-01-0057. The authors thank the referee for suggesting useful modifications.

\section{REFERENCES}

[1] E. Acri and M. Bonatto, Skew braces of size pq, https://arxiv.org/pdf/1908.03228.pdf.

[2] D. Bachiller, Counterexample to a conjecture about braces. J. Algebra 453 (2016), 160-176.

[3] D. Bachiller, F. Cedo, E. Jespers and J. Okninski, Iterated matched products of finite braces and simplicity; new solutions of the Yang-Baxter equation. Trans. Amer. Math. Soc. 370 (2018), 48814907.

[4] W. Bosma, J. Cannon and C. Playoust, The Magma algebra system. I. The user language, Journal of Symbolic Computation 24 (1997), 235-265.

[5] F. Cedo, Left braces: solutions of the Yang-Baxter equation. Adv. Group Theory Appl. 5 (2018), 33-90.

[6] F. Cedo, E. Jespers and A. del Rio, Involutive Yang-Baxter groups. Trans. Amer. Math. Soc. 362 (2010), 2541-2558.

[7] F. Cedo, A. Smoktunowicz and L Vendramin, Skew left braces of nilpotenct type. Proc. London Math. Soc. 118 (2019), 1367-1392.

[8] L. N. Childs, Skew braces and the Galois correspondence for Hopf Galois structures. J. Algebra 511 (2018), 270-291.

[9] P. Dehornoy, Set-theoretic solutions of the Yang-Baxter equation, RC-calculus, and Garside germs . Adv. Math. 282 (2015), 93-127.

[10] C. Dietzel, Braces of order $p^{2} q$, https://arxiv.org/abs/1801.06911.

[11] V. Drinfeld, On some unsolved problems in quantum group theory. Quantum groups (Leningrad, 1990), Lecture Notes in Math. 1510, Springer, Berlin, 1992, pp. 1-8.

[12] B. Eick, H. U. Besche and E. O'Brien, SmallGrp - A GAP package, version 1.3 (2018). (https://www.gap-system.org/Packages/smallgrp.html)

[13] P. Etingof, T. Schedler and A. Soloviev, Set-theoretical solutions to the quantum Yang-Baxter equation. Duke Math. J. 100 (1999), 169-209.

[14] The GAP Group, Groups Algorithms and Programming, version 4.10.2 (2019). ( http://www.gap-system.org)

[15] L. Guarnieri and L. Vendramin, Skew braces and the Yang-Baxter equation. Math. Comp. 86 (2017), 2519-2534.

[16] T. Gateva-Ivanova, Set-theoretic solutions of the Yang-Baxter equation, braces and symmetric groups. Adv. Math. 338 (2018), 649-701.

[17] K. Nejabati Zenouz, Skew braces and Hopf-Galois structures of Heisenberg type. J. Algebra 524 (2019), $187-225$.

[18] W. Rump, Braces, radical rings and the quantum Yang-Baxter equations, J. Algebra 307 (2007), 153-170.

[19] W. Rump, Classification of cyclic braces, II. Trans. Amer. Math. Soc. 372 (2019), 305-328.

[20] A. Smoktunowicz, On Engel groups, nilpotent groups, rings, braces and the Yang-Baxter equation. Trans. Amer. Math. Soc. 370 (2018), 6535-6564.

[21] L. Vendramin, Extensions of set-theoretic solutions of the Yang-Baxter equation and a conjecture of Gateva-Ivanova. J. Pure Appl. Algebra 220 (2016), 2064-2076.

[22] L. Vendramin, Problems on skew braces, Adv. Group Theory Appl. 7 (2019), 15-37.

Sobolev Institute of Mathematics, pr. ak. Koptyuga 4, Novosibirsk, 630090, Russia and Novosibirsk State University, Novosibirsk, 630090, Russia

E-mail address: bardakov@math.nsc.ru

Sobolev Institute of Mathematics, PR. AK. Koptyuga 4, Novosibirsk, 630090, Russia And Novosibirsk State University, Novosibirsk, 630090, Russia

E-mail address: neshch@math.nsc.ru

Harish-Chandra Research Institute, HBNi, Chhatnag Road, Jhunsi, Allahabad-211 019, India

E-mail address: myadav@hri.res.in 